Results 1479 patient underwent a CTC at KGH during the 3year period. 454 patients were aged $\geq 80$ years old $(30.7 \%)$ mean \& median age 84 , range 8-7. 69 patients had positive colonic findings (15.2\% - see figure). Of which, 31 had CRC reported (14 operated straight away, 5 had endoscopy then surgery, 1 had endoscopy only (no tumour seen), 11 were not operated on). 22 had polyps reported (13 had endoscopy at which 12 had polypectomy, 9 did not have endoscopy). 16 had indeterminate findings (10 had endoscopy which nil significant found).

Of the 385 patients who had CTCs with nil significant colonic findings, 9 had extra colonic tumours. At follow up (105 patients in 12 months from October 2015, 137 patients in 201-7, 143 patients in 201-8, range $0-36$ months), none of the patients have been diagnosed with CRC thus far.

Conclusion In this study, CT colonography was used in elderly patients aged $\geq 80$ years old as a first or second line colonic examination. The yield of diagnosing colorectal cancer was $6.9 \%(31 / 454)$. CTC that reported negatively for colonic findings seems to protect patients for -6 months.

Whilst colonoscopy may be the preferred diagnostic test for colonic disease, it is an invasive test with a small risk of perforation. CTC is safer, and better tolerated. The reports are generally accurate with regards to significant colonic findings, especially when diagnosing CRC. This study does confirm the safety and efficacy of CTC and suggests that it is an appropriate colonic investigation for elderly patients (aged $\geq 80$ years old) first or second line.

\section{PWE-061 YOUNG-ONSET COLORECTAL CANCER PRESENTATION IN A DGH POPULATION COMPARED TO OLDER-ONSET PATIENTS}

${ }^{1}$ Siyao Xing*, 'Suzanne Pomfret, ${ }^{1} J o n a t h a n$ Landy, ${ }^{2}$ Drostan Cheetham, 'Susan Catnach. ${ }^{1}$ Gastroenterology Dept, West Hertfordshire Hospitals NHS Trust, Watford General Hospital, Watford, UK; ${ }^{2}$ Department of Colorectal Surgery, West Hertfordshire Hospitals NHS Trust, Watford General Hospital, Watford, UK

\subsection{6/gutjin-2019-BSGAbstracts.385}

Background and Aims The incidence of colorectal cancer (CRC) in patients under 50 years of age is increasing although it is still a rare diagnosis in this age group. However, data suggest young-onset CRC patients have a delayed presentation with higher rates of advanced disease stage at diagnosis. Diagnostic algorithms that increase the specificity of investigations for symptomatic patients in this age group are needed. We aimed to assess features of presentation in young-onset CRC patients in our population and to compare features at presentation with older onset patients.

Methods The trust cancer database was reviewed for all patients with a diagnosis of CRC between May 2015 and 2018. Patients diagnosed under the age of 50 were included in the young-onset group. Older onset CRC patients were matched $4: 1$ by gender and site of tumour (right colon/left colon/rectum). Electronic records were evaluated for presentation and laboratory parameters at diagnosis. Fisher's exact test was used for statistical analysis; odds ratios and confidence intervals were calculated where statistically significant $(\alpha 0.05)$ differences between groups were identified. Data was analysed using GraphPad Prism 5.

Results 43 patients with a diagnosis of CRC aged less than 50 were identified ( $6 \%$ of all CRC diagnoses). 56\% were male with a median age of 40 (IQR 3-6). The most common symptoms at presentation were constipation or diarrhoea (44\%), pain (42\%) and rectal bleeding (37\%). 26\% had $\geq 2$ symptoms at presentation. None had a documented significant family history of CRC. In young-onset CRC patients, $49 \%$ of tumours were in the left colon, $28 \%$ in the right colon and $23 \%$ in the rectum. $37 \%$ were emergency presentations compared with $26 \%$ in the older onset CRC group (ns). $56 \%$ presented with Stage 3 or 4 disease compared with $49 \%$ in patients $\geq 50$ years (ns). 1-year survival was $93 \%$ in youngonset CRC patients $\mathrm{v} 86 \%$ in patients $\geq 50$ years (ns). $51 \%$ of young-onset CRC v $31 \%$ in patients $\geq 50$ years were anaemic at presentation $(p=0.02$; OR $2.395 \%$ CI $1 .-.5)$. $26 \%$ of young-onset CRC v $10 \%$ in patients $\geq 50$ years had elevated platelet counts at diagnosis $(\mathrm{p}=0.02$; OR 2.9 95\% CI 1.3 6.8).

Conclusion In our population young-onset CRC patients there was a trend towards higher rates of emergency presentation and advanced disease stage, as well as a higher 1-year survival rates, although these were not statistically significant compared to older onset CRC patients. A significantly higher proportion of young-onset CRC patients were found to be anaemic and or have elevated platelet counts at diagnosis. Elevated platelet count as well as blood haemoglobin should be investigated further leading to possible incorporation into diagnostic algorithms that might increase the specificity of investigation for symptomatic patients in this age group.

\section{PWE-062 A RETROSPECTIVE REVIEW OF COLO-RECTAL CANCER IN YOUNG ADULTS}

Umair Kamran*, Abdullah Abbasi, Mohsin Zia Sana Ullah, Helen Wright, Arooj Abid, Arun Kurup. University Hospitals of North midlands, Stoke on trent, UK

\subsection{6/gutjnl-2019-BSGAbstracts.386}

Introduction The aim of this study was to assess the trends in clinical presentations and anaemia among young adults [age < 50] with colorectal cancer at the time of diagnosis.

Methods This was a retrospective observational study. The identity of young adults diagnosed with colorectal cancer between 200-017 were obtained from the Somerset cancer data base. Clinical information about the patient was obtained from the electronic patient database [I portal] Descriptive statistics and survival outcomes were performed using SPSS software

Results 171 patients were identified over a period of 8 years (102 males vs 69 females) with median age of 46 years. Prior to diagnosis, the mean duration of symptoms for men was 99 days and for women 91 days. The majority of both male and female patients presented with rectal bleeding $(53.7 \%$ and $46.3 \%$ respectively). Abdominal pain was more common among patients diagnosed with right sided colon cancer compared to the left, $41.2 \%$ vs $20.2 \%, \mathrm{p}=0.0046$ [95\% CI $6.1-$ 6.01]. Almost half $(49.02 \%)$ of the patients were anaemic at the time of diagnosis. Incidence of anaemia was significantly higher in right sided cancers as compared to left colon cancers $[74 \%$ vs $40 \%$ respectively $\mathrm{p}=0.0001]$. Microcytic anaemia [mcv < 80] was seen in $25.2 \%$ patients. Post hoc analysis showed that MCV was significantly lower in patients with cancer in the right colon, compared to those with it in the rectum $(p=0.01)$. Rectum was the most common site of the tumour (47.95\%) and 73\% (116) of patients were diagnosed at advanced stage (stage III/IV). 
Conclusions Incidence of colorectal cancer in young adults is increasing $^{1}$ and they tend to present at advanced stage. Our study showed that most patients were symptomatic for at least 3 months prior to diagnosis. Although rectal bleeding was the most common presenting complaint, microcytic anaemia and abdominal pain tend to be more common in right sided colon cancer. These findings should alert primary care clinicians and specialists alike when faced with a young adult with gastrointestinal symptoms.

\section{REFERENCES}

1. Siegel, RL. (2017). Colorectal Cancer Incidence Patterns in the United States, 1974-2013. Journal of the National Cancer Institute. American Cancer Society, Atlanta February 2017

\section{Neurogastroenterology}

\section{Orals}

\section{OWE-08 PSYCHOSOCIAL FACTORS ASSOCIATED WITH PAIN IN INFLAMMATORY BOWEL DISEASE: A CROSS-SECTIONAL STUDY}

${ }^{1}$ Louise Sweeney, ${ }^{2}$ Rona Moss-Morris, Wladyslawa Czuber-Dochan11, ${ }^{1}$ Trevor Murrells, ${ }^{1}$ Christine Norton. ${ }^{1}$ Faculty of Nursing, Midwifery and Palliative Care, King's College London, London, UK; ${ }^{2}$ Health Psychology Section, King's College London, London, UK

\subsection{6/gutjnl-2019-BSGAbstracts.387}

Introduction Pain is frequently reported by patients with inflammatory bowel disease (IBD). Pain in IBD is not fully explained by disease activity or other clinical findings, and a recent systematic review suggested that psychosocial factors have an important role in IBD-pain. The aim of this study was to investigate psychosocial factors associated with pain in IBD.

Methods 297 adults (>16 years) with IBD were recruited from outpatient clinics $(n=114)$ and online $(n=183)$. Participants completed validated questionnaires assessing pain and potential emotional, cognitive and behavioural correlates. Socio-demographic and clinical factors including disease activity were also recorded.

Results $243(81.8 \%)$ of participants reported pain. Of these 243, mean age was 36 years; 153 (63\%) had Crohn's disease (CD), 90 (37\%) had ulcerative colitis (UC), and 165 (67.9\%) were female. $62.6 \%$ reported mild, $31.6 \%$ moderate and $5.8 \%$ severe pain. Mean disease activity was 7.52 for CD (Harvey Bradshaw Index: HBI) and 5.6 for UC (Simple Clinical Colitis Activity Index: SCCAI), indicating mild to moderate active disease. $40.3 \%$ of participants met established criteria for "chronic pain" and $18.5 \%$ reported opioid use. Female gender, smoking status, prior surgery and steroid use were associated with greater pain severity. Psychosocial factors associated with pain severity and interference included depression, catastrophising, fear avoidance and lower self-efficacy and psychological well-being (Table 1). Regression models explained $44.7 \%$ of the variance in pain severity and $48.6 \%$ of pain interference, with psychosocial factors explaining 9.7\% and $23.5 \%$ respectively when controlling for demographic and clinical variables.
Conclusions Pain in IBD is significantly associated with cognitive and behavioural factors as well as low mood. This study widens a biopsychosocial understanding of pain in IBD and identifies important areas for treatment targets in future interventions.

Abstract OWE-08 Table 1. Correlations between pain and psychological and clinical factors

\begin{tabular}{lll}
\hline & Pain Severity & Pain interference \\
\hline Age & .109 & .050 \\
Faecal calprotectin $(\mathbf{n}=43)$ & -.069 & -.072 \\
HBI & $.558^{* *}$ & $.532^{* *}$ \\
SCCAI & $.482^{* *}$ & $.379^{* *}$ \\
IBS Severity Score & $.664^{* *}$ & $.614^{* *}$ \\
Pain Self-Efficacy & $-.457^{* *}$ & $-.592^{* *}$ \\
Pain Catastrophising & $.453^{* *}$ & $.506^{* *}$ \\
Fear Avoidance & $.356^{* *}$ & $.415^{* *}$ \\
Symptom Focusing & $.267^{*}$ & $.374^{* *}$ \\
Embarrassment Avoidance & $.379^{* *}$ & $.453^{* *}$ \\
All or Nothing Behaviour & $.222^{* *}$ & $.331^{* *}$ \\
Damage Beliefs & $.309^{* *}$ & $.337^{* *}$ \\
Avoidance resting behaviour & $.328^{* *}$ & $.478^{* *}$ \\
Pain acceptance & $-.139^{*}$ & -.020 \\
Depression & $.433^{* *}$ & $.507^{* *}$ \\
Anxiety & $.254^{* *}$ & $.367^{* *}$ \\
Stress & $.250^{* *}$ & $.357^{* *}$ \\
Psychological well-being & $-.372^{* *}$ & $-.391^{* *}$ \\
\hline
\end{tabular}

\section{OWE-09 OUTCOMES OF GUT-FOCUSED HYPNOTHERAPY IN SCHOOL CHILDREN AND ADOLESCENTS WITH SEVERE REFRACTORY IRRITABLE BOWEL SYNDROME}

Syed S Hasan, Pamela Cruickshanks, Peter J Whorwell, Dipesh H Vasant* Neurogastroenterology, Wythenshawe Hospital, Manchester University Foundation Trust, Manchester, UK

\subsection{6/gutjnl-2019-BSGAbstracts.388}

Introduction Irritable Bowel Syndrome (IBS) symptoms in school children and adolescents are relatively common, often causing considerable embarrassment, social withdrawal, disruption to education and psychological distress. Pharmacological options in this group are limited due to safety concerns concerning antidepressants, making behavioural therapies such as gut-focussed hypnotherapy an attractive treatment option. Here, we evaluated outcomes from gut-focussed hypnotherapy in patients with IBS $\leq 18$ years of age.

Methods Consecutive young patients ( $\leq 18$ years of age) with severe IBS symptoms referred to our tertiary neurogastroenterology unit received 12 sessions of gut-focussed hypnotherapy at weekly intervals using the Manchester Protocol. All patients completed the Tellgen Absorption Scale before treatment and all completed the following measures before and after hypnotherapy; IBS symptom severity score (IBS-SSS), Hospital Anxiety and Depression scale (HADS), Non Colonic Symptom score and quality-of-life (QoL) score. Interference with education and the impact of hypnotherapy was also recorded. The primary outcome measure was response to hypnotherapy 